\title{
Small satellites beyond boundaries
}

\section{P. Tortora ${ }^{1}$}

Published online: 18 September 2020

(c) The Author(s) 2020
It is a great pleasure for me to present this special issue of the CEAS Space Journal devoted to Small Satellites Beyond Boundaries. This brings together some of the most interesting presentations delivered throughout several conferences held in the last couple of years in the area of Small Satellites and CubeSats.

Small satellites (and, more recently, CubeSats) were initially developed for educational purposes, however their capabilities have opened a new design space for exploration that includes stand-alone spacecraft, constellations, and mother-daughter systems that collaborate. In light of recent advances in science instruments and spacecraft miniaturization technologies that have emerged in just the past few years, small spacecraft can now be considered for use in planetary exploration, either as adjuncts to larger missions on which they could "catch a ride" to the most remote and challenging destinations in the solar system, or in some cases, as stand-alone missions of their own. The first ever experience of an interplanetary mission carried out using CubeSats is the Mars Cube One (MarCO) mission to Mars, flown as a companion to the InSight mission to Mars. Next, a spectacular set of 13 CubeSats will be delivered in 2021 to a high lunar orbit within the frame of NASA's Artemis-1 mission-the first launch of their new space launch system (SLS) rocket.

Can small satellites expand the boundaries of our knowledge and be the key element of the next era of solar system exploration and deep space observation?

In the seventies the great advancement of solar system exploration was carried out through relatively large and heavy missions like Voyagers and Pioneers, which turned out to be unbelievably reliable, but at a large price in terms of mission cost and development time. In the nineties the

P. Tortora

paolo.tortora@unibo.it

1 Dipartimento di Ingegneria Industriale, Interdepartmental Center for Industrial Research in Aerospace (CIRI-AERO), Alma Mater Studiorum-Università di Bologna, Forlì (FC), Italy flagship missions which followed up (like Galileo and Cassini) were even larger, more complex and expensive than their predecessors, but accomplished wonderful enterprises at their target Jupiter and Saturn planetary systems. We are now at a turning point, where smaller systems can accomplish missions unthinkable just a few years ago. Small satellites have dramatically reduced the cost of Earth observation and they are on the course of making deep space exploration and observation missions more affordable.

The first paper by Freeman presents an overview of the technological challenges and the new opportunities offered by the first pair of interplanetary CubeSats launched to Mars to provide a relay capability for the InSight lander back to Earth. In addition, this paper provides an overview of other CubeSat mission developments at JPL, in particular two CubeSats that are currently under development and that will form part of the 'swarm' of CubeSats escorted onto a lunar trajectory by NASA's Artemis-1 mission.

The next two papers report about in-flight experience from on-going missions. The paper by Eriksen et al., describes the in-flight experience of two Norwegian small satellites, NorSat-1 and NorSat-2, launched in July 2017, equipped with the latest space automatic identification system (AIS) receiver. The manuscript investigates the improvements made globally as well as in selected areas in terms of the number of messages received and number of ships detected. It also quantifies the performance increase through three types of developments: the receiver electronics and the algorithms, the antenna diversity, and the multichannel architecture giving continuous operation on all four AIS channels. The paper by Gordon et al. describes the first mission by Technische Universität Berlin, named TechnoSat, making use of their novel modular TUBiX20 microsatellite platform. The manuscript describes also the upgrade of the TUBiX20 platform according to the requirements of the TUBIN project, an Earth Observation mission scheduled for launch in 2020. Here the focus is set on illustrating how the modular platform design allows scaling the performance while at the same time keeping the modifications to the flight-proven system at a minimum. 
The following pair of papers deals with Missions and Systems Analysis for future missions. The paper by Sarno et al. introduces a strategy for an autonomous reconfiguration of a fractionated synthetic aperture radar (SAR) spacecraft system where the radar antenna is distributed on a smallsatellite formation. The following paper by Devani et al. describes the system design of a $6 \mathrm{U}$ CubeSat capable of hosting a "cold atom sensor". The consortium have built and vibration tested a CubeSat prototype meeting the requirement of cooling atoms down to 100s of micro kelvin, as a pre-cursor to gravity sensors for future Earth observation missions.

Next paper by Bruhn et al., discusses current implementations and roadmap for leveraging high performance computing tools and methods on small satellites with radiation tolerant hardware. This feature can be very beneficial to those missions carrying modern sensors that can generate very large amounts of data such as optical sensing, hyperspectral, synthetic aperture radar (SAR), and Infrared imaging. In addition, a $1 \frac{1}{2}$ CubeSat volume unit cloud computing solution based on AMD $28 \mathrm{~nm}$ APU technology is presented as an example of heterogeneous computer solution.

The following paper by von der Ohe analyses the problem of small satellite telecommunication frequency allocations below $1 \mathrm{GHz}$, in an era when hundreds of new small satellites are being launched each year, and the RF spectrum for satellite communication becomes increasingly occupied. The International Telecommunication Union recognized this problem at World Radiocommunication Conference in 2015 and formed several study groups. This paper presents the results of the study groups and the regulatory measures proposed to satisfy the small satellite developers' needs.

The next four papers deal with several aspects of Nanosats/CubeSats/PocketQubes. The first one by Bouwmeester et al. describes the characteristics of PocketQubes in terms of their constraints and their potential utility, with a focus on space debris, collision risks, and the orbital decay characteristics. Due to technical constraints, such as form factor, power and attitude control, the domain of applications for single PocketQube missions is limited, but they still can be used as low-cost training and technology demonstration platforms. The second one by Byrne et al. presents compact S-band and X-band antenna products dedicated to CubeSat applications and their radio frequency operations. Their remarkable performance and small form factor, together with the strong space heritage of the used processes and materials, make these antennas perfectly suited for professional CubeSat missions with high level of performance and reliability. The last two papers deal with CubeSat Guidance Navigation and Control Systems. The one by Modenini et al. describes the development and experimental validation of an automatic mass balancing system for a dynamic CubeSat attitude simulator. The system is meant to reduce significantly the time necessary for tuning the platform and to minimize the residual gravity torque, expected to be the largest among the disturbances. The experimental results show the effectiveness of the implemented approach, which leads to a residual disturbance torque acting on the balanced platform smaller than $5 \times 10^{-5} \mathrm{Nm}$. The last paper by Kuiper et al. presents an ADCS system design tailored for $6 \mathrm{U}$ CubeSats for Earth observation. The goal is to reach sub-meter spatial resolution at $230-380 \mathrm{~km}$ altitude, and this requires a careful preliminary design of the attitude actuators (a momentum wheel and magnetorquers), which performance was assessed trough numerical simulations.

In summary, the areas of technology development to allow small satellites to be employed beyond the boundaries of LEO orbits are very wide. Small satellites have the undeniable advantage of being cheaper to build than traditional larger spacecraft, but their use in deep space requires more durable components than their commercial counterparts in LEO. In addition, the costs stemming from spacecraft operations must be carefully addressed as, to first order, they do not scale with spacecraft size and may then represent a nonnegligible portion of the budget of a deep space mission based on a small satellite platform.

CEAS Associate Editor on Small Satellites: P. Tortora.

Open Access This article is licensed under a Creative Commons Attribution 4.0 International License, which permits use, sharing, adaptation, distribution and reproduction in any medium or format, as long as you give appropriate credit to the original author(s) and the source, provide a link to the Creative Commons licence, and indicate if changes were made. The images or other third party material in this article are included in the article's Creative Commons licence, unless indicated otherwise in a credit line to the material. If material is not included in the article's Creative Commons licence and your intended use is not permitted by statutory regulation or exceeds the permitted use, you will need to obtain permission directly from the copyright holder. To view a copy of this licence, visit http://creativecommons.org/licenses/by/4.0/.

Publisher's Note Springer Nature remains neutral with regard to jurisdictional claims in published maps and institutional affiliations. 